\title{
Construcción cultural de la sexualidad en un grupo de mujeres. Implicaciones para el ámbito de los cuidados
}

\author{
Cultural construction of sexuality in a group of \\ women. Implications for the field of care \\ Construção cultural da sexualidade em um grupo de \\ mulheres. Implicações para o campo do cuidado
}

\author{
José Hernández - Ascanio ${ }^{1}$, Pedro Ventura Puertos ${ }^{2}$ \\ ${ }^{1}$ Profesor, Universidad de Córdoba. Correo electrónico: jhascanio@gmail.com \\ 2 Profesor, Universidad de Córdoba. Correo electrónico: pventuura@uco.es \\ Cómo citar este artículo en edición digital: Hernández-Ascanio, J., \& Ventura Puertos, P. (2019). \\ Construcción cultural de la sexualidad en un grupo de mujeres. Implicaciones para el ámbito de los \\ cuidados. Cultura de los Cuidados (Edición digital), 23 (54). Recuperado de \\ http://dx.doi.org/10.14198/cuid.2019.54.25
}

Correspondencia: Dpto. de Ciencias Sociales y Humanidades; Facultad de Filosofía y Letras; Universidad de Córdoba; Plaza del Cardenal Salazar, n. 5, Córdoba

Correo electrónico de contacto: jhascanio@uco.es

Recibido: 10/12/2018; Aceptado: 18/05/2019

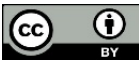

\begin{abstract}
Despite the importance of sexuality in the life trajectory of any individual, this has been a traditionally invisible issue, something especially evident and restrictive in the case of women, in whom the issue of sexuality has usually been a space of control and of resistance.

The absence of studies on sexuality is significant in disciplines related to care, which have shown a late interest in this issue, despite being recognized as a space conditioning health and well-being. When it has been approached it has been done from a fundamentally biomedical perspective, without taking into account the conditioning role of socio - cultural constructions on it, a question that has limited the intervention capacity of health
\end{abstract}

professionals.

This work, of a qualitative nature carried out through in-depth interviews and discussion groups with rural women in the province of Córdoba, aims to address this identified gap, as well as highlighting the main constructs on sexuality with which women manage their day to day, with the purpose of providing care professionals with useful tools for their professional performance in this particular field.

Keywords: Sexuality, nursing care, gender, sex education.

\section{RESUMO}

A ausência de estudos sobre sexualidade é significativa nas disciplinas relacionadas ao cuidado, que têm demonstrado um interesse tardio nessa questão, apesar de ser 
reconhecida como espaço condicionante de saúde e bem-estar. Quando abordado, foi feito a partir de uma perspectiva fundamentalmente biomédica, sem levar em conta o papel condicionante das construções socioculturais, questão que tem limitado a capacidade de intervenção dos profissionais de saúde. Este trabalho, de natureza qualitativa realizado em profundidade entrevistas e discussões em grupo com mulheres rurais na província de Córdoba, pretende abordar essa lacuna identificada, bem como para destacar o que as principais construções da sexualidade com mulheres gerenciar o seu dia a dia, com o objetivo de fornecer aos profissionais de saúde ferramentas úteis para o seu desempenho profissional neste campo particular.

Palavras chave: Sexualidade, cuidados de enfermagem, gênero, educação sexual.

\section{RESUMEN}

Pese a la importancia de la sexualidad en la trayectoria vital de cualquier individuo, ésta ha sido una cuestión tradicionalmente invisibilizada, algo especialmente evidente $y$ taxativo en el caso de las mujeres, en quienes la cuestión de la sexualidad ha sido normalmente un espacio de control y de resistencia. La ausencia de estudios sobre sexualidad es significativa en disciplinas relacionadas con el cuidado, que han demostrado un tardío interés por esta cuestión, pese a ser reconocido como un espacio condicionador de la salud y el bienestar integral. Cuando ha sido abordado se ha hecho desde una perspectiva fundamentalmente biomédica, sin tener en cuenta el papel condicionante que ejercen las construcciones socio - culturales sobre la misma, cuestión que ha limitado la capacidad de intervención de los profesionales sanitarios. Este trabajo, de carácter cualitativo llevado a cabo mediante entrevistas en profundidad y grupos de discusión con mujeres rurales de la provincia de Córdoba, pretende abordar ese vacío identificado, así como poner de manifiesto cuáles son los principales constructos sobre sexualidad con los que las mujeres gestionan su día a día, con la finalidad de aportar a los profesionales del cuidado herramientas útiles para su desempeño profesional en este ámbito en concreto.

Palabras clave: Sexualidad, cuidados enfermeros, género, educación sexual.

\section{INTRODUCCIÓN}

Pese a que el ámbito de la sexualidad es una cuestión de especial interés como foco de intervención en cuidados es una temática sobre la que apenas se dispone de investigaciones y de conocimiento contrastado, y muy especialmente, en el caso de las mujeres. En la mayoría de las ocasiones, el planteamiento dominante es el de carácter biomédico, que considera la sexualidad como algo intrínseco, natural, universal y biológicamente determinado, planteando que el deseo femenino disminuye de manera natural con la menopausia (Buss, 1998).

Esta postura choca frontalmente con la propia definición integral que la Organización Mundial de la Salud (OMS, 1975) hace de la sexualidad, incluyendo dimensiones de carácter psicosocial, con implicaciones económicas, políticas, culturales, éticas, legales, históricas, religiosas y espirituales que interpelan al individuo y que ha sido puesto de manifiesto en estudios llevados a cabo sobre esta temática (Freixas y Luque, 2009; Freixas, Luque y Reina, 2015; Wood, Mansfield y Kock, 2007). 
La dedicación de una mayor atención a la salud sexual y el control de la reproducción ha ido adquiriendo un creciente interés en sus múltiples dimensiones, siempre de la mano de iniciativas sociales comprometidas con la agenda de los movimientos de mujeres y del feminismo de la nueva ola en el mundo (Giddens, 2000). Este interés creciente ha trascendido al ámbito de la incidencia pública a través de su reconocimiento en diferentes acuerdos internacionales, tales como las Conferencias Internacionales de Desarrollo, Derechos Humanos y Población, la Convención sobre la Eliminación de Todas las Formas de Discriminación contra la Mujer (CEDAW), la Plataforma de Acción de la Conferencia de El Cairo sobre Población y Desarrollo o la IV Conferencia Mundial sobre la Mujer, celebrada en Beijing en 1995.

De un análisis de marcos de estos documentos, se podría inferir que la salud sexual y reproductiva entraña capacidades de disfrutar de una vida sexual satisfactoria y sin riesgos, así como la asunción de la maternidad como una opción libre $y$ planificada. Pese a que de manera teórica es posible identificar una cierta simetría entre la importancia dada al ámbito de la sexualidad procreativa y la sexualidad recreativa, en la práctica hay un mayor énfasis en el primer aspecto, evidenciándose un claro déficit en cuanto a la formulación de contextos y de programas de atención que abarquen la sexualidad de las mujeres en su dimensión más amplia, pues, con frecuencia, los proyectos que se ponen en marcha tanto desde las instancias gubernamentales como desde la sociedad civil, dejan fuera el ámbito de la sexualidad recreativa, lo que tiene efectos tangibles en cómo las mujeres gestionan ese ámbito concreto de sus vidas.

En suma, parecería que no es importante considerar las características particulares de la sexualidad recreativa de las mujeres para establecer los criterios de atención en el ámbito de la salud sexual. Sin embargo, de las construcciones que sobre la sexualidad hacen las mujeres se van a derivar cuestiones tan importantes como la toma de decisiones acerca de la gestión de su salud sexual y reproductiva, el abordaje de la maternidad o el afrontamiento de relaciones distópicas de género, por poner algunos ejemplos. La realidad es que existen pocas investigaciones que partan de la premisa de identificar las construcciones culturales y psicosociales que contextualizan este ámbito. Por ello, este trabajo pretende cubrir este déficit abordando las principales construcciones que sobre la sexualidad hacen mujeres del medio rural para ponerlo en relación con las implicaciones en la prestación de cuidados que de las mismas se puede derivar.

Los trabajos considerados hasta el momento han ido aportando de manera progresiva elementos de carácter conceptual que han ido enriqueciendo el debate sobre la temática propuesta, pero han adolecido de un sesgo claramente directivo $y$ homogeneizador en la mayoría de los casos, y en los menos, una orientación claramente experiencial y subjetiva. Hasta tal punto ha ocurrido esto, que nos encontramos con que la sexualidad es un constructo que profundamente encastrado en la dimensión de género, que posteriormente se ha ido matizando con otras variables como la orientación sexual o la edad. Sin embargo, en muy pocas ocasiones ha sido posible encontrar aportes que situaran la sexualidad en un mismo plano de igualdad que el concepto de género, como elementos que se matizan y reelaboran mutuamente, y que, por tanto, son igualmente productos socialmente construidos y culturalmente 
transmitidos. Aportar esa perspectiva nos puede ayudar a comprender en buena medida muchas de las tensiones $y$ distorsiones que las mujeres pueden manifestar al tener que lidiar con ese proceso de incorporación y reelaboración que no siempre son fáciles ni coherentes con otros aspectos de su construcción bio - psico - socio - cultural como individuos.

En este trabajo partimos de identificar las construcciones que hacen las propias mujeres en base a su experiencia compartida pero también al despliegue de recursos de carácter personal, de forma que se puedan identificar los elementos transversales que son comunes en torno a variables como la edad, la formación o la experiencia de residencia y que forman parte de su matriz identitaria como individuos.

\section{METODOLOGÍA}

Se apostó por un diseño de investigación cualitativa de teoría fundamentada, dentro del paradigma del interaccionismo simbólico, básicamente, por las siguientes razones: Es evidente el déficit de conocimiento que existe acerca de esta temática $\mathrm{y}$, de ahí, la necesidad de saber cómo lo conciben actrices sociales particularmente expuestas a la misma. En segundo lugar, desde una óptica de cuidados, la sexualidad es un objeto de estudio bastante apropiado para un abordaje cualitativo, puesto que se enmarca en la dimensión subjetiva de la realidad social. No queríamos analizar únicamente la sexualidad de la mujer como un hecho social, sino interpretando el significado que le otorgan ellas mismas.

El ámbito de realización se enmarcó en la Comarca del Alto Guadalquivir Cordobés en la provincia de Córdoba, España. Como criterios de inclusión se tuvieron en cuenta mujeres mayores de edad, con competencia comunicativa y social para poder participar de entrevistas en profundidad y grupos de discusión, naturales y residentes en algunos de los municipios que componen dicha comarca.

Previa a la selección de las participantes, se llevó a cabo un muestreo teórico que tuvo en cuenta una tipología de actrices sociales implicadas en el objeto de estudio, con una variedad significativa de experiencias, y donde la adscripción generacional, la formación académica y la orientación sexual fueron fundamentales. La selección se hizo mediante estrategia de bola de nieve utilizando como facilitadoras las asociaciones y colectivos de mujeres presentes en cada municipio, así como las trabajadoras del Centro de Información a la Mujer comarcal.

En cuanto a la recogida de datos, se realizó entre marzo y agosto de 2017, usando las instalaciones municipales y recurriendo a la combinación de entrevistas en profundidad y grupos de discusión:

- Las entrevistas sumaron un total de 73, con una duración media de 45 minutos, $\mathrm{y}$ derivaron de las preguntas de investigación iniciales (¿cómo definen las mujeres la sexualidad? ¿con qué componentes la asocian? ¿a partir de qué contextos construyen esas definiciones? ¿hay una variabilidad en relación a la edad?).

- En cambio, los grupos de discusión fueron 4 con 5 participantes cada uno, contaron con la figura de un observador, y tuvieron una duración media de 60 minutos.

La estrategia de análisis estuvo orientada por el enfoque de propio de la Teoría Fundamentada (Glaser y Strauss 1967), 
recurriendo al programa NVivo 9 para la operativización de dicho proceso. Se siguió una dinámica de codificación abierta (Stake, 1995; Simons, 2011), que permitió además descubrir subcategorías y categorías significativas. Luego se pasó a agrupar esa información por temas, mediante el método de comparaciones constantes (Strauss y Corbin 2002).

Los principios fundamentales de la investigación biomédica fueron tenidos en cuenta en todo momento por el conjunto de autores, informando de los objetivos y garantizando a los sujetos del estudio el anonimato y la confidencialidad de los datos, recogidos con su permiso expreso. El análisis de los datos se hizo de forma desagregada, con codificación de doble ciego, en relación a las informantes que participaron. Para su exposición, se recurrió a una nomenclaura basada en la combinación de una letra mayúscula (asignada aleatoriamente a cada una de las participantes), una cifra de dos dígitos (correspondiente con la edad de la informante) y una abreviatura relativa al nivel de estudios académicos.

\section{RESULTADOS Y DISCUSIÓN}

Respecto a los resultados, se analizaron los discursos de las entrevistas y grupos de discusión, identificando los campos semánticos que utilizaban las mujeres al elaborar su propia definición de lo que pudiera ser la sexualidad, con palabras significativas o con metáforas e imágenes representativas.
Como se puede ver claramente, los resultados obtenidos ponen de manifiesto que la sexualidad no es un elemento sólido, biológicamente acotado y determinado, sino que es un espacio en continua construcción y reelaboración. Dicho proceso se da en diálogo y a veces en contestación, con espacios sociales y culturales que pretenden normativizar la sexualidad en términos claramente afines a un modelo patriarcal. La sexualidad es un campo de conquista y de resistencia de muchas mujeres, que articula su identidad como sujetos teniendo profundos efectos en su salud psicosocial. Los mecanismos tradicionales de control sobre esta esfera de la mujer tienen que ver con el control de la información, la transmisión de determinadas normas y valores, así como la aplicación de una experiencia de "disonancia experiencial" mediante la cual se desarticula la sexualidad de una concepción integral del sujeto. En este proceso, elementos como la variable edad y nivel educativo van a ser especialmente importantes, porque suponen la posibilidad de acceder o no a determinados recursos con los que resistir a esa estrategia de control que hemos comentado anteriormente.

Una vez hechas estas puntualizaciones, pasamos a analizar con más detalle algunas de las categorías que emergieron durante el proceso de investigación y que reflejan los principales aspectos de nuestro marco explicativo (Figura 1). 


\section{FIGURA 1: Marco explicativo del estudio}

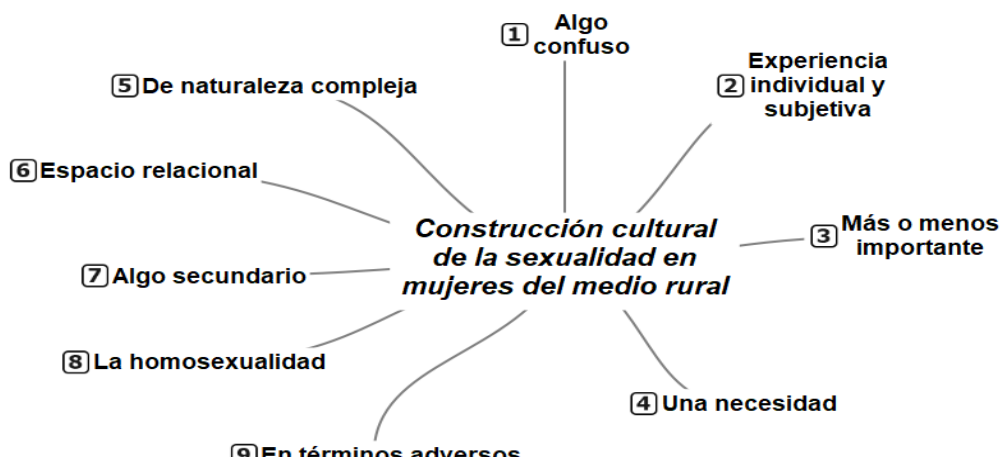

FUENTE: Elaboración propia

\section{La sexualidad como algo confuso}

En primer lugar, la sexualidad es algo "confuso", muchas veces "indeterminado", que se construye por la concurrencia de otros conceptos o ideas que les resultan más operativas. Frente a algunas de estas palabras evocadoras que fueron surgiendo (tales como amor, sexo, cariño, deseo o erotismo), descubrimos que la sexualidad es un concepto que ocupa un segundo nivel de abstracción sobre el cual hay poca reflexión objetiva por parte de las mujeres, así como que es más una experiencia pragmática experiencial en la que, para hablar de ella, se precisa de un tiempo previo de reflexión antes de formular ideas. Estas indefiniciones (cuando menos definiciones abiertas) hacen tremendamente difícil poder establecer diálogos fluidos que no estén conectados a hechos concretos.

No sabría definírtelo, así me pillas un poco en blanco, me imagino que toda la actividad que tenga una connotación erótica, no tiene por qué estar vinculada con el amor siempre, y yo lo veo como algo muy natural que ha estado rodeado de tabúes, prejuicios y nos han vuelto locos porque todo giraba en torno a lo mismo y que habría que vivirla con otra naturalidad, aunque no somos capaces de transmitirlo a nuestros hijos que se diga con naturalidad, con naturalidad aunque con cierta sensatez aunque muchas veces la sensatez con la sexualidad no tiene nada que ver, pero a ver como se lo dices a tu hija cuando tiene catorce años. Yo cuando lo pienso, lo pienso siempre con otra persona intuitivamente, no significa que no lo contemple como algo personal, tu contigo misma, yo te soy sincera, yo cuando lo pienso intuitivamente lo pienso con otra persona.

$$
\mathrm{H}-48 \text { - UNIV }
$$

Primero creo que es algo muy abierto, primero porque creo que la sexualidad empieza desde que somos pequeños, primero porque es algo humano y es algo del cuerpo que se estimula desde que somos pequeños y luego cuando somos mayores, es algo que está en nuestras vidas y que va desde una caricia o un abrazo también puede tener un aspecto sexual hasta lo que es algo mas como es el coito, creo que es algo muy amplio, no sabría definirlo, pero creo que sería algo así...por una parte creo que físicamente es mío pero creo que con mi pareja o también puede ser, no sé, con mi pareja es en conjunto.

$$
\text { F - 28 - UNIV }
$$


La verdad es que nunca me lo he planteado, es una difícil pregunta, no sé, por un lado, se me viene a la cabeza sexo, pero no es solo eso, es todo el conjunto, es algo que nunca me he parado a pensar, no sé, lo relaciono tal vez con placer, claro que sí, no sabría cómo decirlo.

\section{D - 24 - ESTMED2}

Durante un largo periodo de tiempo, la sexualidad ha estado fuertemente vinculada con otros conceptos como el amor por ejemplo en el imaginario colectivo, teniendo como espacio preferencial de representación el ámbito del matrimonio. Sin embargo, hay saltos generacionales que permiten visibilizar como los procesos de "destradicionalización" (Beck et al, 1994) junto con una mayor tolerancia social hacia las relaciones sexuales han permitido desbordar la sexualidad de los límites del matrimonio y de su vinculación con el amor en las generaciones más jóvenes.

\section{La sexualidad como una experiencia individual y subjetiva}

Relacionado con la idea anteriormente expuesta, está la justificación de que, intuitivamente, la sexualidad es una experiencia profundamente íntima $\mathrm{y}$, por lo tanto, difícilmente comunicable y dependiente estructuralmente de la experiencia vital de cada sujeto. El sexo o el amor aparecen como "conceptos duros", entendido esto como que tienen una entidad lo suficiente sólida como para poder ser considerados en sí mismos sin necesidad de tener que hacer referencia a otros. Además, parte de esa solidez de concepto viene determinado por la percepción de que es algo "común y universal", con independencia de la expresión histórica, cultural o geográfica que puedan tener. Sin embargo, la sexualidad como concepto no parece tener esa solidez, lo que podría ser explicada en parte por el hecho de que sería como resultado de la combinación flexible de otros varios elementos, y esto ya cae en la esfera de lo estrictamente individual y subjetivo. Una imagen que nos ayudaría a entender esto sería las de las figuras de un "mecano", en las que todos los sujetos parten de un número similar de piezas pero tienen libertad (la realidad de esta libertad será discutida más adelante) para escoger y combinar las mismas según sus gustos, intereses o necesidades.

Depende de cada persona, hay gente más echada y otros no tanto, puede ser que se sientan atraídos por ti, atracción puede ser.

$$
\text { B - } 39 \text { - ESTMED }
$$

Abarca muchas cosas, desde pareja a orientación. Es todo lo que tiene que ver con el tema de pareja, de sexo, en este momento. Lo relaciono con el placer por ejemplo.

$$
\text { B - } 28 \text { - UNIV }
$$

\section{La sexualidad en términos de importancia}

Un primer bloque de definiciones serían las que abordan la sexualidad en términos de "importancia" en términos absolutos, pero en las que descubrimos que la sexualidad es entendida como la consecución lógica o la prolongación de un "algo". Es decir, si no se da la primera premisa (la existencia o disponibilidad de una pareja, por ejemplo) la segunda, que sería la expresión de la sexualidad, se vería innecesaria o algo totalmente marginal en la vida de la persona. Especialmente significativo es este discurso en mujeres que ya cuentan con una edad avanzada, no disponen de compañero /a íntimo /a en este momento o se ven condicionadas por las condiciones de salud de dichas parejas.

No le doy mucha importancia a la sexualidad. Sexualidad hay muchas clases, porque yo que 
sé, porque yo por ejemplo con que me tocaban un poquito, ahora no me toca nadie, pero cuando me tocaban un poquito te ponías y a lo mejor era muy bonito que hacer otras cosas, otras se piensas que es hacerlo y ya está... yo ahora sigo viviendo mi sexualidad a mi modo, sola... una cosa es sexo y otra cosa es sexualidad...

\section{B - 77 - ESTBAS}

Es algo que tiene poco sentido porque mi pareja tiene un problema de salud, además de otras cuestiones que hacen que sea imposible que aquello se ponga en plan, yo le doy menos importancia de la que tiene, y hay otras cosas con las que puedes jugar sin necesidad de, yo he tenido orgasmos sin necesidad de penetración y yo por eso no le voy a querer menos, ni él va a ser menos hombre... si me pilla bien, disfruto el momento...

$$
\text { B - } 55 \text { - ESTMED }
$$

\section{La sexualidad como "una necesidad"}

Un segundo bloque son las que "naturalizan" la sexualidad de tal forma que se constituyen en "necesidades vitales básicas como beber o comer". Estas construcciones conceptuales coinciden en justificar, en primer plano, un origen biológico a la sexualidad y, en un segundo, en ser uno de los elementos estructurales de la relación con otras personas, especialmente en el contexto concreto de la pareja.

Esta naturalización de la sexualidad puede dotar de argumentos a los comportamientos que se generan en torno a ella (con independencia del efecto beneficioso pernicioso de los mismos), así como servir de justificación para una llamada de atención sobre la necesidad de dar visibilidad con una mayor "normalización" a esta temática. Especialmente interesante resulta la naturalización realizada como elemento constitutivo de la pareja (o de vínculo análogo entre sujetos) sin el cual es imposible pensar a dicho vínculo o, cuando menos, lo condiciona de una forma extremadamente importante.

Es una cosa de la naturaleza que el cuerpo, que en la etapa de joven si yo supiera aquello no hubiera perdido el tiempo que hemos perdido, disfrutar el cuerpo es ley de naturaleza.

$$
\text { B - } 68 \text { - ESTABAS }
$$

Hombre, es importante, hoy por hoy ya es que ya está perdiendo mucho peso, pero necesaria es, no diría ya del 10 como en otro momento, pero que su 4 o 5 todavía lo necesita uno, una rutina, un de vez en cuando sí, eso no puede fallar, no, porque entonces qué clase de pareja es?, para estar, comer, estar juntos nada más, salir de paseo?, tiene que haber algo más?... lo del coito nada más no me afecta, pero la complejidad de tener a alguien de cuando estés tranquila $y$ te llega o que te diga buenos días y te dé un beso, eso sí me destrozaría perderlo, eso sí me destrozaría.

$$
\text { F - } 56 \text { - UNIV }
$$

Disfrutar con alguien o contigo misma y satisfacer tus necesidades, es una necesidad, porque te lo pide el cuerpo, somos animales, a veces la tienes y a veces no, y cuando la tienes es como si tienes hambre, o calmas ese hambre o tienes hambre y te cabreas, es un instinto básico como el del ser humano igual que los animales... es la satisfacción mutua entre las personas de la pareja, ya tiene que ver con otra manera de relacionarse en la pareja.

$$
\text { D - } 46 \text { - UNIV }
$$

\section{La naturaleza compleja de la sexualidad}

Otro conjunto de definiciones son las que mejor ejemplifican la "dimensión compuesta" de la sexualidad, asumiendo un carácter holístico para la misma sin que exista una relación asimétrica entre las diferentes dimensiones a partir de las cuales las informantes construyen su concepto de sexualidad. En estas definiciones vemos 
como la sexualidad adquiere un carácter aglutinante de las dimensiones bio - psico emocionales y de los espacios intra - inter relacionales de la persona.

La sexualidad es que es todo, porque al principio es cuando empiezas de pareja, placer, es placer, es un puro placer, después ya empiezas placer pero con un poquito de cariño de cariñitos, de tonterías, entonces la sexualidad ya se abre a más mimos, más hablar, mas complicidad, cuando vas más mayor ya casi lo que es parte corporal es lo de menos, es más el estar con alguien, poder contar con él, pero claro, no puedes dejar tampoco la parte corporal por mucho que quieres porque entonces no tiene, tiene que ir mezclándose una cosa con otra aunque lo corporal va perdiendo un poquillo de peso, no debes de dejarlo porque si lo dejas pierdes la pareja, persona que se crea con cariño, cuanto te quiero o cuatro besitos va a ser que la pareja no sigue, sin sexualidad no sigue la pareja, pero vamos, no es tampoco que sea todo sexualidad igual a coito, ese concepto de cuando tienes quince o veinte años de sexualidad igual a coito eso ya no, es sexualidad es estar con alguien y sentirte a gusto y conocer tu cuerpo, conocer el suyo, no sé, ¿entiendes?

$$
\text { F - } 56 \text { - UNIV }
$$

Lo asocias a sexo, pero a más cosas, todas las cosas que eso conlleva, las personas de diferentes gustos, de diferentes formas en general... intimidad...

$$
\text { D - } 30 \text { - ESTMED }
$$

Ahondando en la dimensión bio - psico emocional de la sexualidad es posible encontrar que el bienestar físico - mental psicológico es una categoría que aparece de manera recurrente a la hora de elaborar la definición de sexualidad. Es un elemento especialmente valorado por las informantes y pivota sobre las "elaboraciones naturalizadoras" que hemos visto anteriormente. Existe una correlación directa entre entender la sexualidad como "necesidad" y percibir que la cobertura o no de la misma tiene una serie de efectos sobre la integridad de la persona. Es significativo cómo se pone de manifiesto que, salvo raras excepciones, la relación entre necesidad cobertura se establece normalmente en términos de incremento cuanti y cualitativo, es decir, que mayor / mejor cobertura de dicha necesidad mayores / mejores serán los beneficios integrales sobre el sujeto. En muy pocas ocasiones nos hemos encontrado entre las participantes la manifestación de que una ausencia de cobertura conlleva aspectos negativos.

Bienestar, relajación, confianza, todo eso, bienestar y paz, confianza, para mi confianza, lo entiendo como algo dentro y fuera de la pareja, pero yo no te puedo hablar de eso porque sólo he tenido relaciones dentro de la pareja, yo no he tenido relaciones sexuales, yo también empecé muy jovencita, si estuviera sola pues hablaría también de diversión, alegría, algo que necesitas, porque además cuando no lo tienes se nota.

$$
\text { D - } 37 \text { - UNIV }
$$

La sexualidad, sentirte bien contigo mismo o misma, y mostrarte tal y como eres con otra persona, y tener una persona o varias que puedas s sentirte cómoda en ese aspecto de tu vida, que es algo muy importante en la vida de cualquier persona, la satisfacción sexual es algo fundamental... si pasamos mucho tiempo sin hacer nada me influye en el carácter y la relación no fluye de la misma manera.

$$
\text { B - } 34 \text { - UNIV }
$$

Es un tema placentero, a raíz de ahí te puede sacar felicidad, sonrisa, quedarte floja, para mí es bueno, para mi creo que hace falta, habrá personas a las que no les haga falta, pero para mí es necesario, pero yo creo que no les hace falta o no lo necesitan porque no se conocen a sí mismas, sabes, porque el sexo para uno mismo es lo mejor que te puedes encontrar, con una pareja es bonito, vale, pero si no te conoces a ti misma, que vas a hacer con eso?, 
se lo vas a dar a la otra persona para que disfrute él, pero y que pasa contigo?

$$
\text { B - } 28 \text { - ESTBAS }
$$

\section{La sexualidad como espacio relacional}

A continuación, le vamos a prestar especial atención a los espacios relacionales que se le atribuyen a la sexualidad. Vemos cómo la sexualidad se construye en torno al eje de "la sexualidad es un espacio de relación". En el eje de mayor inespecificidad, nos encontraríamos con la sexualidad como experiencia de comunicación contigo misma o con otra persona, mientras que, en el foco más extremo, se entiende como una experiencia de comunicación sujeta a principios de relación y, más concretamente, en el escenario de la pareja.

En el foco más "inespecífico" nos encontramos formulaciones tales como las siguientes:

Es una manera de comunicarte con otra persona, digo con otra persona porque podría ser tanto hombre como mujer, y es una manera de alcanzar placer físico y placer emocional, porque s hay amor también hay placer emocional, si hay amor hay otro tipo de placer físico... para mí la sexualidad es importante pero hoy por hoy no me acostaría con un hombre simplemente por tener sexo, pero si me lo daría yo porque mi cuerpo me lo pide.

$$
\text { D - } 61 \text { - UNIV }
$$

Para mí es compartir, respeto, placer, diversión, intimidad.

$$
\text { E - } 39 \text { - UNIV }
$$

Para mi sexualidad es amor, respeto, autoestima y placer y muchísima muchísima comunicación, pero entiendo que para la gente el componente del amor no sea necesario, pero para mí la sexualidad tiene que tener amor, deseo, pero sobretodo comunicación, respeto, eso a rajatabla.

$$
\text { D - } 26 \text { - UNIV }
$$

Un paso más sobre las formulaciones anteriormente llevadas a cabo es la que sitúa la sexualidad dentro de un vínculo de relación (dirigida a una misma o hacia otra persona) que cuenta con unos códigos normativos más o menos establecidos.

La sexualidad, que entiendo por sexualidad, pues todo lo referente tanto a una pareja como contigo misma, creo, todo lo referente al cuerpo... yo lo que entiendo es eso, referente a yo conmigo misma y con mi cuerpo y también referente a la pareja tal vez, porque otro concepto no lo considero.

$$
\mathrm{H} \text { - 33- UNIV }
$$

Para mí son las relaciones que puedan tener dos personas, ya sean del mismo sexo o no, y las que puedan tener uno mismo consigo mismo.

$$
\mathrm{H} \text { - } 27 \text { - UNIV }
$$

Para mí la sexualidad es el conjunto de hechos que tienen entre una, que tienen en lugar entre dos personas que se atraen, creo yo que es, no solamente el sexo, sino lo que viene siendo la primera mirada, tocamientos entre comillas, el tonteo, hasta que llegue el momento.

\section{D - 21 - ESTMED}

Un grado mayor de concreción en el interfaz comunicación - relación, nos lo encontramos cuando se incorpora la expresión explícita de las relaciones sexuales en dichas experiencias. Es importante tener en cuenta que esta dimensión es incorporada paulatinamente conforme interaccionamos con generaciones más jóvenes, encontrando el punto de inflexión en las mujeres que se encuentran en la generación de los cincuenta años para abajo. Ello es claramente identificable en el discurso de aquellas mujeres que han desarrollado su proceso de socialización durante los últimos cincuenta años, en los que se puede constatar una mayor visibilidad de las relaciones sexuales en todos los ámbitos de la vida social.

Para mí, tal y como lo veo es estar con una 
persona con la que quieres, para mí personalmente, entrar por la puerta un tío que no conoces de nada e irme esa misma noche como que no, tiene que haber algo, algo de química, de conocerte por lo menos de dos o tres dias, tiene que haber algo, mínima amistad, pero tiene que haber algo... yo nunca he tenido de esas experiencias ni yo tampoco las he querido... tampoco me ha dado tiempo porque llevo con mi pareja desde los catorce años.

\section{D - 40 - SINEST}

Hombre, es una pregunta complicada, es dificil porque vamos a ver, sexualidad, sexualidad, yo siempre he referido al sexo $y$ cuando hablo de sexo me refiero al acto sexual de dos personas, dependiente de luego ya de la orientación que tengan, entonces yo sexualidad yo creo que es sobre lo que me vas a preguntar sobre temas referentes al acto sexual, al sexo tuyo, yo que sé....

\section{F - 40 UNIV}

La sexualidad no es solo penetración, es también un abrazo, es un cariño, para mí es más importante, para ellos a lo mejor no, no sé si es porque las mujeres pensamos de otra manera...

$$
\text { D - } 37 \text { - UNIV }
$$

Para mí es la totalidad de la persona, se me ocurre que tiene que ver con el sexo, el respeto de la persona, los detalles que tienes con tu pareja... la sexualidad tiene que ser que te traten con cariño, que te cuiden, no la viviría bien si me la impusieran o si me forzaran.

\section{D - 37 - ESTMED}

Pues ideas, para mí es que nazca un sentimiento entre las dos personas, una atracción física, esa la primera idea, que haya atracción física, palabras que se me ocurren atracción física, amor, amor, deseo, placer, esas son las que yo considero... para sentirme plena debo tener mi sexualidad satisfecha.

$$
\text { D - } 21 \text { - ESTMED }
$$

Pues yo que sé, dos personas que se quieren mucho, es que no sé cómo explicarlo, dos personas que se quieren mucho y que están juntas, es que no sé qué explicar... no creo que sea tampoco una cosa muy, ni que sea como quien dice poca cosa ni muy muy muy importante, una cosa natural, normal, que yo no veo que sea tampoco, tampoco creo que me afectaría tener una vida plena si no tuviera mucho sexo...

$$
\text { D - } 18 \text { - ESTMED }
$$

El mayor grado de focalización a la hora de situar la sexualidad en un entorno de relación es el que lo enmarca en el contexto concreto de la pareja, como estructura formal y estable que actúa como panóptico desde el cual entender al otro y entenderse a sí mismo. Estos discursos son más frecuentes en las generaciones de mujeres más mayores y en aquellas en los que sus itinerarios vitales vienen determinados por un mayor arraigo en los pueblos, así como por un menor nivel de instrucción. En estos casos, es frecuente encontrar una correlación con la constatación de que se dan las mínimas parejas sexuales en sus trayectorias vitales, limitándose en muchas ocasiones a un único caso.

Es un acto de amor, que realizas con una persona a la que quieres, o con una persona que no quieres, según, cada persona, tan hombre como mujer, una forma de demostrar que lo quieres, o que te gusta...

$$
\text { D - } 63 \text { - ESTMED }
$$

Sin sentimiento no tiene sentido para mí, forma parte de un sentimiento, de una relación, es una mezcla de muchas cosas.

$$
\text { D - } 58 \text { - ESTMED }
$$

Algo muy íntimo, muy intimo, con una persona a la que quieres, con alguien con quien tienes feeling en ese momento, que te da placer... es un desahogo del cuerpo que puede ir acompañado o no de amor o de enamoramiento, con el amor como que va más adornado, pero es un desahogo del cuerpo, una necesidad...

$$
\text { D - } 57 \text { - SINEST }
$$

La sexualidad es difícil de explicar, porque según con quien, una cosa es sexo y otra sexualidad, es difícil de explicar, en mi cabeza 
solo cabe de que sea con mi pareja, mi cabeza no da más, tal vez sea por el lugar donde me he criado o bien por el tiempo que hace, porque tú te ibas de aquí y en lo último que pensabas era en volver a casa con un bombo con alguien que no fuese de tu pareja... es algo fundamental...

$$
\text { D - } 50 \text { - SINEST }
$$

Yo que sé, depende de la situación, puede ser echar un rato, otras veces con tu pareja, que tengas tu intimidad, si eres más grande, pues para formar familia. Hombre, ahora mismo pues echar un rato o buscar algo más serio, buscar algo más serio...

$$
\text { D - } 22 \text { - ESTMED }
$$

Frente a este continuo que hemos expresado anteriormente en este trabajo, también nos encontramos con referencias marginales a la sexualidad como algo reducido a su expresión física, especialmente la relacionada con la de relaciones sexuales.

Entiendo dos definiciones, la de aquí llego aquí te pego y ya está, o como la sexualidad para mí después de estar tanto tiempo con pareja, de haber conocido una persona que llevas tanto tiempo con ella pues, pero si, es que también sexualidad te puedes referir a dos personas del mismo sexo, que es el tema que ahora está más, ahora el tema es ese es lo que veo yo.

$$
\text { B - } 32 \text { - ESTMED }
$$

En algún momento si he tenido relaciones puntuales, pero en este momento no porque estoy formal. Tampoco ha sido uno aquí y otro alli, a lo mejor ha sido varias veces con la misma persona, pero de esto nadie se ha enterado, siempre he sido muy discreta, nunca lo he ido contando por ahí porque nadie se tiene que enterar.

$$
\text { D - } 22 \text { - ESTMED }
$$

\section{La sexualidad como algo secundario}

Junto con las construcciones de concepto que hemos podido ver hasta ahora, también nos encontramos con algunas posiciones que intentan conciliar diferentes aspectos de los que hemos visto dentro del concepto, pero que suelen coincidir en adscribir a la sexualidad un carácter secundario en sus vidas.

Un complemento dentro de una vida para sentirte plena, si no no te sientes plena porque tu cuerpo lo necesita, no es el primordial...

D - 45 - ESTMED

\section{La homosexualidad}

Una mención aparte se merece el indagar acerca de si existen diferencias substanciales a la hora de elaborar el concepto sexualidad entre sujetos en función de su orientación sexual. Hasta el momento, hemos recogido la construcción que han hecho mujeres heterosexuales $y$, a continuación, reservamos un lugar especial a mujeres lesbianas. No encontramos diferencias significativas a la hora de situarse ante la sexualidad, salvo por el hecho de sentirse sometidas a un mayor escrutinio social, a una mayor presión familiar hasta que se da el momento de "aceptación" de esa realidad, y a una mayor tensión personal hasta que se asume en términos de diversidad y no de desigualdad esa característica.

Yo no quería salir con uno y luego con otro, yo tenía que era para toda la vida, entonces yo no salía con nadie, salí nada más que un día con uno, pero me daba vergüenza que no le quería, y luego ya me enamoré, yo no me he enamorado de un hombre. El hecho de ser una mujer sí me ha hecho pensarme por qué me ha tocado a mí, por qué yo no puedo vivir como una pareja normal, porque es diferente, a la gente, a la familia, no puedes vivirlo o disfrutar como una pareja que es un hombre, o yo, la culpable soy yo por no haberlo dicho en su momento... es una relación oculta, no lo sabe sólo las personas que son más cercanos, mis padres no lo saben porque para ellos sería un disgusto muy grande, ellos son mayores y su forma de pensar pues no 
es de ese tipo, con sus pensamientos, ojalá pudiera convivir, pero es complicado.

\section{D - 43 - ESTMED}

\section{La sexualidad en términos adversos}

Las construcciones realizadas al respecto son marginales y cuantitativamente poco representativas, pero ejemplifican cómo la elaboración del concepto no es ajena al propio devenir biográfico de la persona, y cómo en torno a malas experiencias pueden desarrollarse conceptos muy negativos de profundo calado.

Yo he llegado a odiar el sexo, es lo que menos echo de menos, llevo 8 años sin tener sexo, no es que sea una cosa que me ha calado, pero seguramente, en aquel momento he llegado a odiar el sexo... el sexo tiene que ser una complicidad, pasarlo bien, olvidarte de lo demás $y$ estar bien en ese momento.

D - 53 - ESTMED

\section{CONCLUSIONES}

Si bien en el ámbito tradicional de los cuidados se ha dado mucha importancia a la dimensión biológica, profiláctica y procreadora de la sexualidad, esta dimensión apenas es reconocida por sus protagonistas, quienes identifican un mayor peso a la dimensión comunicativa $\mathrm{y}$ relacional de dicho espacio. Tanto una postura como otra son simplificaciones del ámbito de la sexualidad, que dificulta una gestión integral de la misma. El desencuentro entre los marcos de partida entre mujeres y profesionales sanitarios también genera que el diseño y la ejecución de las acciones de cuidado por parte de profesionales de la salud (programas de formación, intervenciones, etc) no respondan a las expectativas y necesidades de las mujeres implicadas, lo que se traduce en intervenciones ineficaces.
Un análisis general muestra cómo la sexualidad es percibida por parte de las mujeres principalmente como una manera de expresar el sentimiento amoroso, siguiendo el ideal romántico de la época moderna, y en el caso de no existir este amor, como una forma de conectarse con otra persona de una manera especial y única. Hay una pérdida progresiva de importancia por parte del ideal tradicional de sexualidad relacionado a aspectos clásicos como la procreación.

Es posible identificar cómo el constructo "sexualidad", que utilizan las mujeres participantes de este estudio, está atravesado de manera importante por elementos asociados a los mandatos culturales de género dentro del sistema patriarcal de tal manera que, en la mayoría de las ocasiones, la vivencia de la sexualidad por parte de estas mujeres es una vivencia vicaria; es decir, que viene determinada $\mathrm{y}$ regulada por la presencia de un tercer sujeto, que es la que la legitima y la autoriza.

Con este trabajo se evidencia que la sexualidad de la mujer es un ámbito todavía caracterizado por la abstracción, la indefinición y la invisibilización bajo el argumento de que "forma parte de lo privado", lo que crea un potencial contexto de riesgo y socaba las posibilidades de una atención plena e integral por parte de los profesionales del ámbito de los cuidados.

\section{BIBLIOGRAFÍA}

- Beck, U., Giddens, A. \& Lash, S. (1994). Reflexive modernization. Cambridge: Polity Press.

- Buss, D. M. (1998). Sexual Strategies Theory: Historical Origins and Current Status. The Journal of Sex Research, 35(1), 19-31.

- Fine, M. (1988). Sexuality, schooling, and adolescent females: The missing discourse of desire». Harvard Educational Review, 58(1), 54-63. 
- Freixas, A. (2006). Demà més. Dones, vides $i$ temps. Barcelona: Institut Català de les Dones.

- Freixas, A. \& Luque, B. (2009). El Secreto mejor guardado: la sexualidad de las mujeres mayores. Política y Sociedad, 46(1), 191-203.

- Freixas, A., Luque, B. \& Reina, A. (2015). Sexuality in Older Spanish Women: Voices and Reflections. Journal of Women E Aging, 27, 1-24.

- Gannon, L. (1998). The Impact of Medical and Sexual Politics on Women's Health. Feminism \& Psychology, 8(3), 285-302.

- Giddens, A. (2000). La transformación de la intimidad. Sexualidad, amor y erotismo en las sociedades modernas, Madrid: Cátedra.

- Gómez Cantarino, S. \& Moreno Preciado M. (2012). La expresión de la sexualidad durante la gestación y el puerperio. Cultura de los Cuidados, 16 - 33.

- Hite, S. (1977). El Informe Hite. Estudio de la Sexualidad Femenina. Barcelona: Plaza \& Janés.

- Iglesias de Ussel, J. (1983). La sociología de la sexualidad en España: notas introductorias, REIS, 21,103-133.

- Kinsey, A. C., Pomeroy, W. B., Martin, C. E. \& Gebhard, P. H. (1953/1967). Conducta sexual de la mujer. Tomo I y Tomo II. Buenos Aires: Ediciones Siglo Veinte.

- Masters, W. H. \& Johnson, V. E. (1966). Human sexual response. Boston: Little Brown.

- Mcneill, P., Freeman, B. \& Newman, J. (1992/1995). Las mujeres hablan del sexo. Barcelona: Serres.

- Osborne, R. \& Guasch, O. (2003) Sociología de la sexualidad, Madrid, CIS.

- Plummer, K. (1991). La diversidad sexual. Una perspectiva sociológica, en Nieto, J. A. (Coord.); La sexualidad en la sociedad contemporánea. Lecturas antropológicas, Madrid, Fundación UniversidadEmpresa.

- Rich, A. (1980/2001). Heterosexualidad obligatoria y existencia lesbiana en Rich, A. (Ed.), Sangre, pan y poesía. Prosa escogida 1979-1985 (pp. 41-86). Barcelona: Icaria.

- Simons, H. (2011), El estudio de caso. Teoría y Práctica. Madrid, Ed. Morata.

- Stake, R. E. (1995), Investigación con estudios de casos, Madrid, Ed. Morata.

- Strauss, A. \& Corbin, J. (2002), Bases de la Investigación Cualitativa. Técnicas y procedimientos para desarrollar la teoría fundamentada. Antioquia, Ed. Universidad de Antioquía.

- Walz, T. (2002). Crones, Dirty Old Men, Sexy Seniors: Representations of the Sexuality of Older Persons. Journal of Aging and Identity, 7(2), 99-112.

- Wood, J. M., Kock, P. B., \& Mansfield, P. K. (2006). Women's sexual desire: a feminist critique. Journal of Sex Research, 43(9), 236-244.

- Wood, J. M., Mansfield, P. K. \& y Kock, P. B. (2007). Negotiating Sexual Agency: Postmenopausal Women's Meaning and Experience of Sexual Desire. Qualitative Health Research, 17(2), 189-200. 